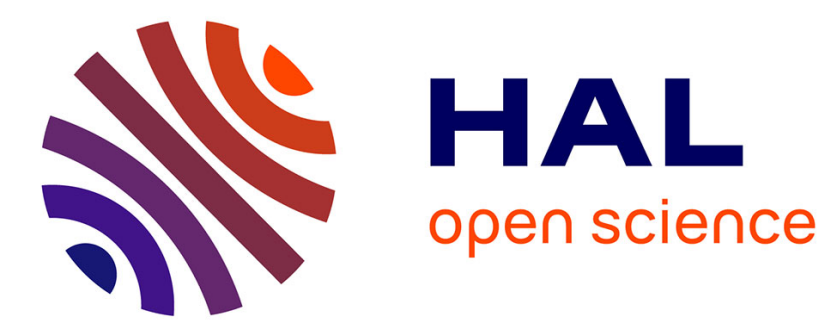

\title{
Neurodegeneration in tauopathies and synucleinopathies
}

Clovis Foguem, Bernard Kamsu-Foguem

\section{To cite this version:}

Clovis Foguem, Bernard Kamsu-Foguem. Neurodegeneration in tauopathies and synucleinopathies. Revue Neurologique, 2016, 172 (11), pp.709-714. 10.1016/j.neurol.2016.05.002 . hal-01635627

\section{HAL Id: hal-01635627 https://hal.science/hal-01635627}

Submitted on 17 Nov 2017

HAL is a multi-disciplinary open access archive for the deposit and dissemination of scientific research documents, whether they are published or not. The documents may come from teaching and research institutions in France or abroad, or from public or private research centers.
L'archive ouverte pluridisciplinaire HAL, est destinée au dépôt et à la diffusion de documents scientifiques de niveau recherche, publiés ou non, émanant des établissements d'enseignement et de recherche français ou étrangers, des laboratoires publics ou privés. 


\section{Open Archive Toulouse Archive Ouverte (OATAO)}

OATAO is an open access repository that collects the work of Toulouse researchers and makes it freely available over the web where possible.

This is an author-deposited version published in: http://oatao.univ-toulouse.fr/ Eprints ID: 17837

To link to this article: $\quad$ DOI:10.1016/j.neurol.2016.05.002.

URL : https://doi.org/10.1016/j.neurol.2016.05.002

\section{To cite this version:}

Foguem, Clovis and Kamsu-Foguem, Bernard Neurodegeneration in tauopathies and synucleinopathies. (2016) Revue Neurologique, vol. 172 (N¹1). pp. 709-714. ISSN 0035-3787 


\title{
Short communication \\ Neurodegeneration in tauopathies and synucleinopathies
}

\author{
C. Foguem ${ }^{a, b}$, B. Kamsu-Foguem ${ }^{c, *}$ \\ ${ }^{a}$ Epernay Hospital Centre, 137, rue de l'Hôpital Auban-Moët, BP 137, 51205 Epernay, France \\ ${ }^{\mathrm{b}}$ Center for Food and Taste sciences (CSGA), UMR 6265 CNRS, UMR 1324 INRA, University of Burgundy, 9E, \\ boulevard Jeanne-d'Arc, 21000 Dijon, France \\ ' Laboratory of Production Engineering (LGP), EA 1905, ENIT-INPT University of Toulouse, 47, avenue d'Azereix, \\ BP 1629, 65016 Tarbes cedex, France
}

Keywords:

Neurodegenerative diseases

Alzheimer's disease

Parkinson's disease

Dementia with Lewy bodies

Dementia

\begin{abstract}
A B S T R A C T
While increasing life expectancy is a major achievement, the global aging of societies raises a number of medical issues, such as the development of age-related disorders, including neurodegenerative diseases. The three main disease groups constituting the majority of neurodegenerative diseases are tauopathies, alpha-synucleinopathies and diseases due to repetitions of glutamine (including Huntington's disease). In each neurodegenerative disease, the accumulation of one or more aggregated proteins has been identified as the molecular signature of the disease (as seen, for example, in Alzheimer's disease, Parkinson's disease, dementia with Lewy bodies, amyotrophic lateral sclerosis and frontotemporal dementia). The etiology of neurodegenerative diseases is often multifactorial, and the known risk factors include, in addition to genetic polymorphisms and age, some other possible causes, such as certain immune and metabolic conditions, endocrine pathologies, gender, socioeconomic or professional status, oxidative stress or inflammation, vitamin deficiencies and environmental factors (chemical exposure, metals). However, innovative strategies to elaborate suitable diagnostic and therapeutic approaches (aiming to at least delay or possibly even reverse disease progression) require further knowledge of the genetic and adaptive immunological characteristics of neurodegenerative diseases.
\end{abstract}

\section{Introduction}

In every neurodegenerative disease, the accumulation of one or more aggregated proteins has been identified as the molecular signature of the disease [as seen in, for example, Alzheimer's disease (AD), Parkinson's disease (PD), dementia with Lewy bodies (DLB), amyotrophic lateral sclerosis (ALS) and frontotemporal dementia (FTD)] [1]. The proteins that accumulate in the central nervous system of patients with such diseases were initially identified by the purification of polypeptides from the brains of affected humans and animals [2]. It is currently assumed that the basic mechanism of cell death in degenerative diseases is related to a rather restricted number of processes in which oxidative stress

\footnotetext{
* Corresponding author.

E-mail address: bernard.kamsu-foguem@enit.fr (B. Kamsu-Foguem).
} 
could play a central role, resulting in protein dysfunction and aggregation, which may be signs of differential clinical expressions [3].

The identification of specific mutations in certain genes is oriented towards certain proteins and pathways currently considered critical in the pathogenesis of the most prominent neurodegenerative diseases (Table 1). These include mutations of precursor proteins: amyloid beta ( $A \beta$ or Abeta) causes $\mathrm{AD}$; alpha-synuclein leads to PD or DLB; and microtubuleassociated protein tau (MAPT) causes FTD with parkinsonism. These aberrant misfolded proteins occurred initially in specific brain areas and were secondarily found in wider areas of the brain as the neurodegenerative disease symptoms worsened or the disease entered its later stages (Figs. 1 and 2) $[4,5]$.

Furthermore, the prion concept explains how a disease can manifest as hereditary, sporadic or infectious. In addition, the common feature of prion diseases (sporadic, dominant inheritance, acquired through infection) is that they involve aberrant metabolism of prion protein. An important feature that distinguishes prion viruses is the two isoforms of prion protein (PrP) encoded by a chromosomal gene. In humans, the PrP gene is designated PRNP and is located in the short arm of chromosome 20.

\section{Table 1 - Intraneuronal filamentous inclusions in neurodegenerative diseases.}

\section{Disease}

Alzheimer's disease

Pick's disease (type of frontotemporal dementia)

FTDP-17

Progressive supranuclear palsy (PSP)

Corticobasal degeneration (CBD)

Parkinson's disease

Dementia with Lewy bodies (DLB)

Multiple system atrophy (MSA)

Huntington's disease

Spinocerebellar ataxia type 1 (SCA1)

Spinocerebellar ataxia type 3 (SCA3)

Spinocerebellar ataxia type 7 (SCA7)

DRPLA, or Naito-Oyanagi disease

Spinal and bulbar muscular

atrophy (SBMA)

FTDP-17: frontotemporal dementia and parkinsonism linked to chromosome 17; PolyQ: polyglutamine; DRPLA: dentatorubral-pallidoluysian atrophy.
Filamentous inclusions

Neurofibrillary tangles (lesions)

Pick bodies

Neurofibrillary lesions, glial fibrillary lesions Neurofibrillary lesions, glial fibrillary lesions Neurofibrillary lesions, glial fibrillary lesions Lewy bodies and neurites

Lewy bodies and neurites Neuronal and glial inclusions

Intranuclear inclusions, dystrophic neurites Intranuclear inclusions

Intranuclear inclusions Intranuclear inclusions Intranuclear inclusions Intranuclear inclusions
Main component

Tau protein

Tau protein

Tau protein

Tau protein

Tau protein

Alpha-synuclein

Alpha-synuclein

Alpha-synuclein

PolyQ repeat expansion in huntingtin protein PolyQ repeat expansion in ataxia type 1 protein PolyQ repeat expansion in ataxia type 3 protein PolyQ repeat expansion in ataxia type 7 protein PolyQ repeat expansion in ataxia type 1 protein PolyQ repeat expansion in androgen receptor

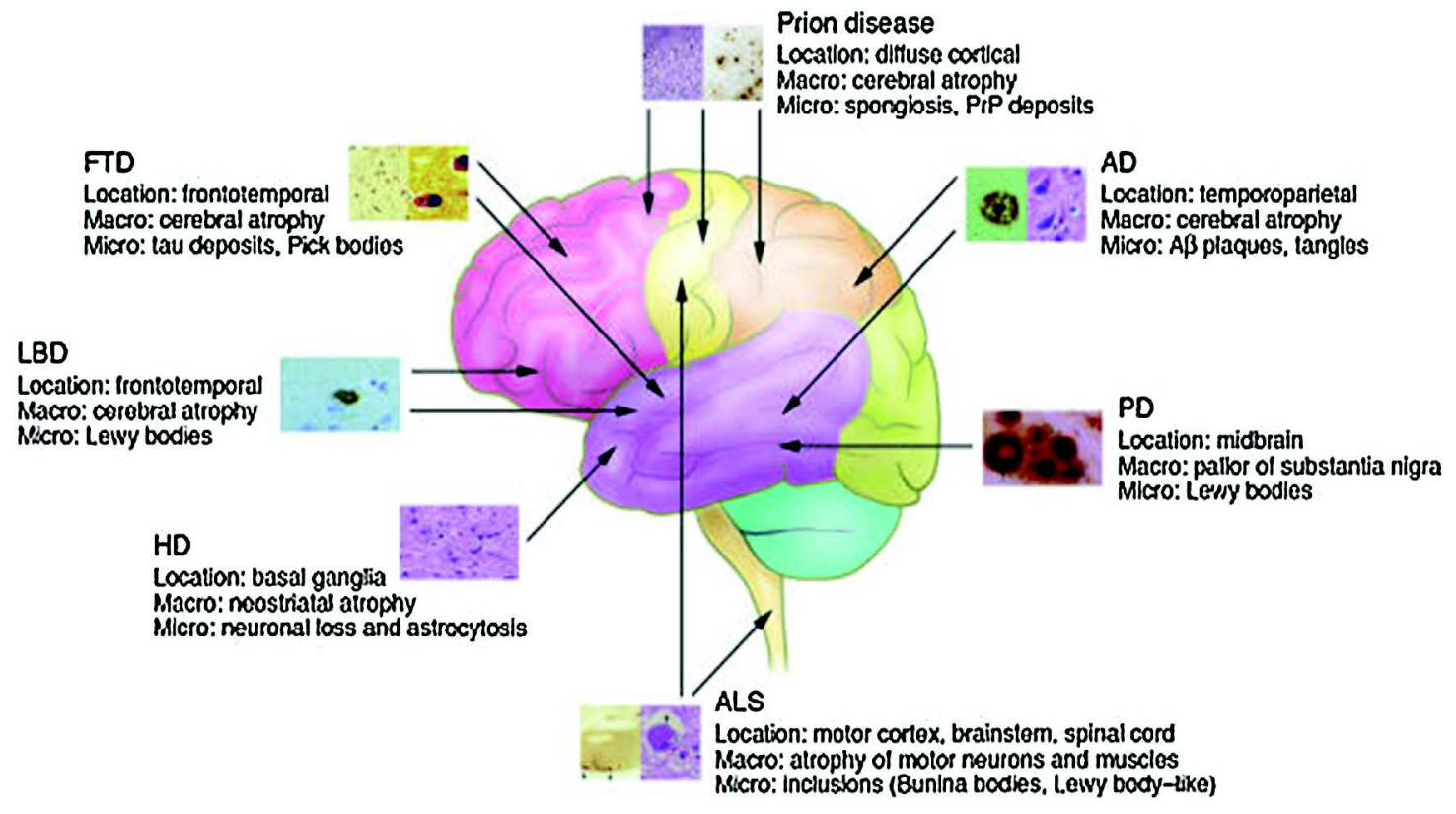

Fig. 1 - Brain anatomical locations of primary macro- and microscopic changes (misfolded proteins) are characteristic of particular neurodegenerative diseases. PrP: prion protein; FTD: frontotemporal dementia; AD: Alzheimer's disease; LBD: Lewy body dementia (including Parkinson disease dementia, PDD and dementia with Lewy bodies, DLB); PD: Parkinson's disease; HD: Huntington's disease; ALS: amyotrophic lateral sclerosis. From Bertram and Tanzi [4]. 

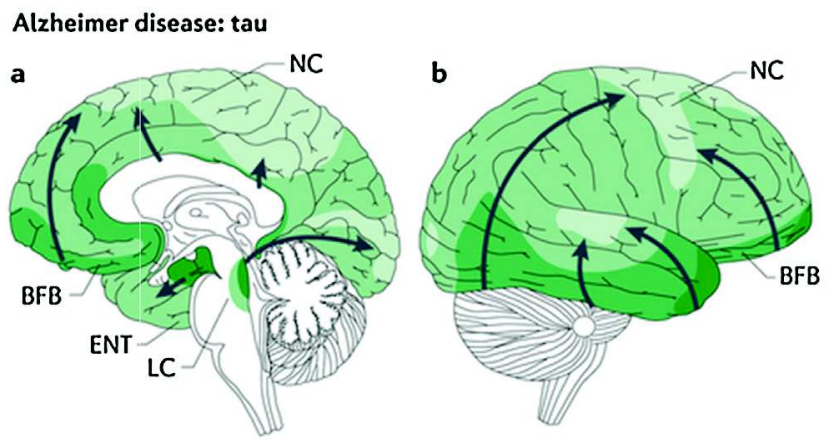

Alzheimer disease: amyloid- $\beta$

Parkinson disease: $a-$ synuclein
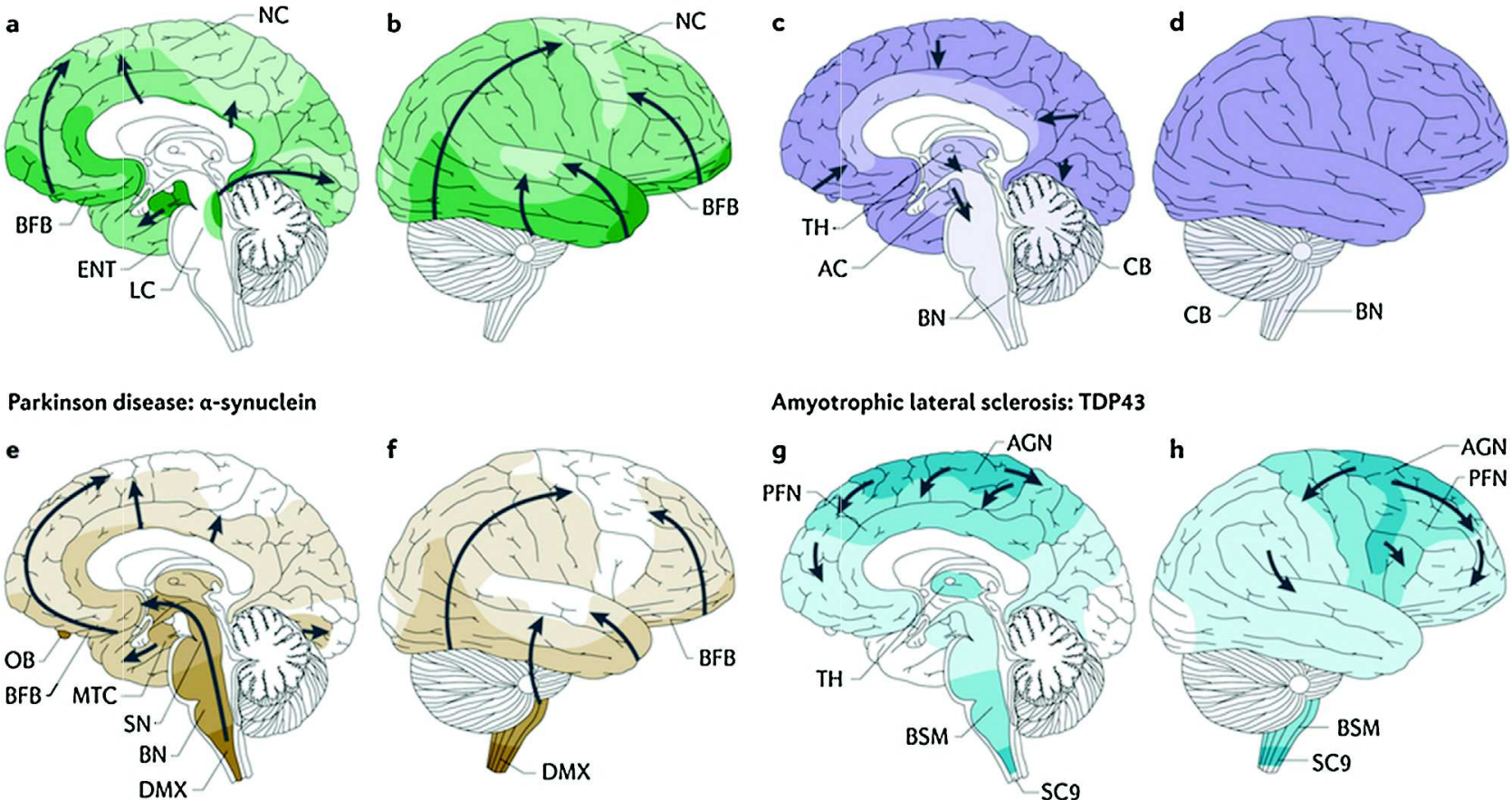

Amyotrophic lateral sclerosis: TDP43
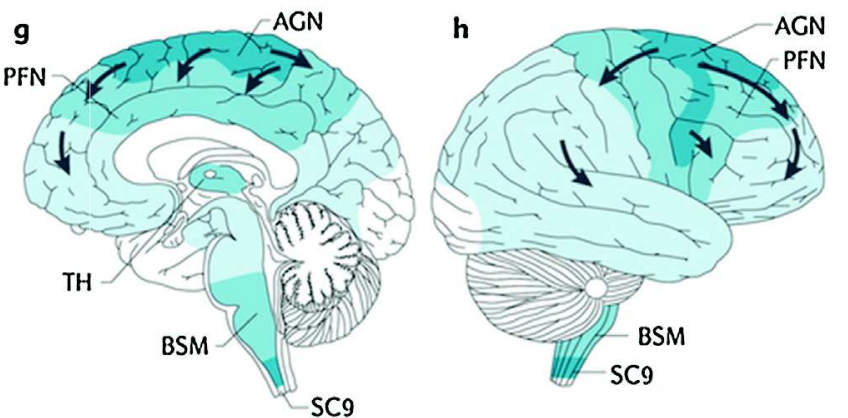

Fig. 2 - Sequential topographical dissemination of non-prion proteins in neurodegenerative diseases. Pathology is first detected in areas shown in darker colours and then in regions shown in lighter colours. ( $a$ and b) In Alzheimer's disease (AD), tau aggregates develop initially in the locus ceruleus (LC), then in transentorhinal and entorhinal regions, and finally in the hippocampal formation and wide areas of neocortex (NC). (c and d) In contrast to tau pathology, amyloid- $\beta$ deposits in $\mathrm{AD}$ are first seen in NC and then in allocortical, diencephalic and basal ganglia structures (caudal direction), brainstem, and sometimes cerebellum (CB). (e and f) Progression of $\alpha$-synuclein immunoreactive Lewy body/neurite pathology in Parkinson's disease follows an ascending pattern from brainstem to telencephalon. Earliest lesions are in the olfactory bulb (OB) and dorsal motor nucleus of the vagus nerve (DMX) in the medulla oblongata. ( $\mathrm{g}$ and $\mathrm{h}$ ) In amyotrophic lateral sclerosis with a low burden of TAR DNA-binding protein 43 (TDP43) pathology, TDP43 inclusions are seen initially in the agranular motor cortex (AGN), brainstem motor nuclei of cranial nerves XII-X, VII and V, and $\alpha$-motor neurons in the spinal cord. AC: allocortex; BFB: basal forebrain; BN: brainstem nuclei; BSM: brainstem somatomotor nuclei; ENT: entorhinal cortex; MTC: mesiotemporal cortex; PFN: prefrontal neocortex; SC9: spinal cord gray-matter lamina IX; SN: substantia nigra; TH: thalamus. Reprinted from Brettschneider et al. [5] and Braak et al. [16].

\section{Filamentous inclusions in neurodegenerative diseases}

The three main disease groups constituting the majority of neurodegenerative diseases are the tauopathies, alpha-synucleinopathies and diseases due to repetitions of glutamine (including Huntington's disease; Table 1).

Tauopathies (including AD, FTD and progressive supranuclear palsy) and alpha-synucleinopathies [comprising mainly $\mathrm{PD}, \mathrm{DLB}$ and multiple system atrophy (MSA)] represent the majority of late-onset neurodegenerative diseases in humans. Amyloidopathies, tauopathies and synucleinopathies share common pathological features in terms of structural, aggregatory and spreading properties (the pathophysiological molecular mechanisms leading to cellular toxicity) [6]. The factors that generate tauopathy and its extension within areas of the brain are not well known; amyloid precursor protein (APP) gene dysfunction is the best candidate so far, as revealed by genetic studies [7]. The development of intracellular filamentous inclusions could bring a risk of acute or chronic toxicity, leading to injury or damage to brain cells [8].

\section{Etiology of neurodegenerative diseases}

Another feature observed in the most common neurodegenerative diseases is the dichotomy between familial (rare) and non-familial forms (most common; Table 2). These are also frequently described as 'sporadic' or 'idiopathic', although there is a growing body of evidence suggesting that a large proportion of these cases are also strongly influenced by genetic factors. The genetics behind these diseases has been labeled 'complex', in contrast to the classical Mendelian genetics dubbed 'simplex' (single mode or single inheritance), due to the involvement of numerous genes that are likely to have complex interactions with each other and with nongenetic variables as well (Fig. 3). The genetics considered complex would be governed by common deoxyribonucleic 
Table 2 - Etiological frequency (sporadic, genetic and infectious) of neurodegenerative pathologies.

\begin{tabular}{lcll} 
Diseases & \multicolumn{3}{c}{ Etiological frequency (\%) } \\
\cline { 2 - 4 } & Sporadic & Genetic & Infectious \\
\hline Prion disease & 85 & $>10$ & $<1$ \\
Alzheimer's disease & 90 & 10 & \\
Parkinson's disease & 95 & $<5$ & \\
Frontotemporal dementia & 90 & 10 & \\
Pick's disease & 95 & $<5$ & \\
Progressive supranuclear & 95 & $<5$ & \\
$\quad$ palsy (PSP) & & & \\
Amyotrophic lateral & 90 & 10 & \\
$\quad$ sclerosis (ALS) & & & \\
Huntington's disease (HD) & & 100 & \\
Spinocerebellar ataxia (SCA) & & 100 &
\end{tabular}

acid (DNA) variants, such as single nucleotide polymorphisms, which significantly increase the risk of disease, yet are insufficient to effectively cause a disorder or specific disease.

Innovative strategies to elaborate suitable therapeutic approaches (aiming to at least delay and possibly even reverse disease progression) require knowledge of the genetic and adaptive immunological characteristics of these neurodegenerative diseases. Genetic evidence supports the idea that multiple neurodegenerative pathways lead to mild cognitive impairment and that the eventual conversion to dementia, principally of the $\mathrm{AD}$ subtype, is essentially due to the $\mathrm{AD}$ pathway(s) [9].

The etiology of neurodegenerative diseases is often multifactorial, although these chronic diseases share two main characteristics:

- neuronal loss in motor, sensory or cognitive systems, generating mental and motor decline;

- a strong association between metabolic changes and neurodegeneration (for example, disruption of the link between the peripheral organs governing energy metabolism and the cognitive and neural systems) [10].

The known risk factors for neurodegenerative diseases include, in addition to genetic polymorphisms and age, other possible causes such as certain endocrine pathologies, gender, socioeconomic or professional status, oxidative stress or inflammation, vitamin deficiencies [especially of vitamin B9 (folic acid) and vitamin B12], immune and metabolic conditions, and environmental factors (such as chemical exposure and heavy metals; Fig. 3). Indeed, some studies have revealed correlations between chronic pesticide exposures in occupational settings and the development of neurological disorders [11]. In addition, epigenetic regulation of gene expression and environmental modulation are factors that may play a key role in the onset and course of common neurological conditions, including $\mathrm{AD}, \mathrm{PD}$, Huntington's disease (HD) and multiple sclerosis (MS) [12]. The immune system also has an important role in neurodegenerative disorders: while there are undoubtedly maladaptive components of neural inflammation that serve to spread a neurotoxic environment that can encourage neuropathogenic lesions, there is also the opportunity to use adaptive and beneficial microglial/monocyte phenotypes to enable beneficial neuroimmune responses in some neurodegenerative diseases (such as $\mathrm{AD}$ and $\mathrm{PD}$ ) [13].

\section{Discussion}

The degenerative processes in brain cells are considered 'primary' when no causal factor can be identified, and 'secondary' when triggering factors are demonstrated (inflammatory factors, or toxic, metabolic or traumatic events). These triggering factors of neurodegenerative pathologies are still mostly unknown, making a timely and accurate diagnosis difficult to achieve during antemortem assessment of these diseases [1]. Although in certain cases, the diagnosis made by a clinician can be confirmed postmortem by pathological examination, it is generally accepted that diagnostic errors may be identified from the patient's postmortem anatomical pathological findings. In many situations, there are difficulties in diagnosis, as some neurodegenerative diseases have many signs and symptoms in common. The diagnosis of DLB is particularly difficult as some of its symptoms can be found in other neurodegenerative diseases, such as $\mathrm{PD}$ and $\mathrm{AD}$, thereby often leading to its misdiagnosis.

\section{COMMON NEURODEGENERATIVE DISEASES IN THE ELDERLY: KEY POINTS}

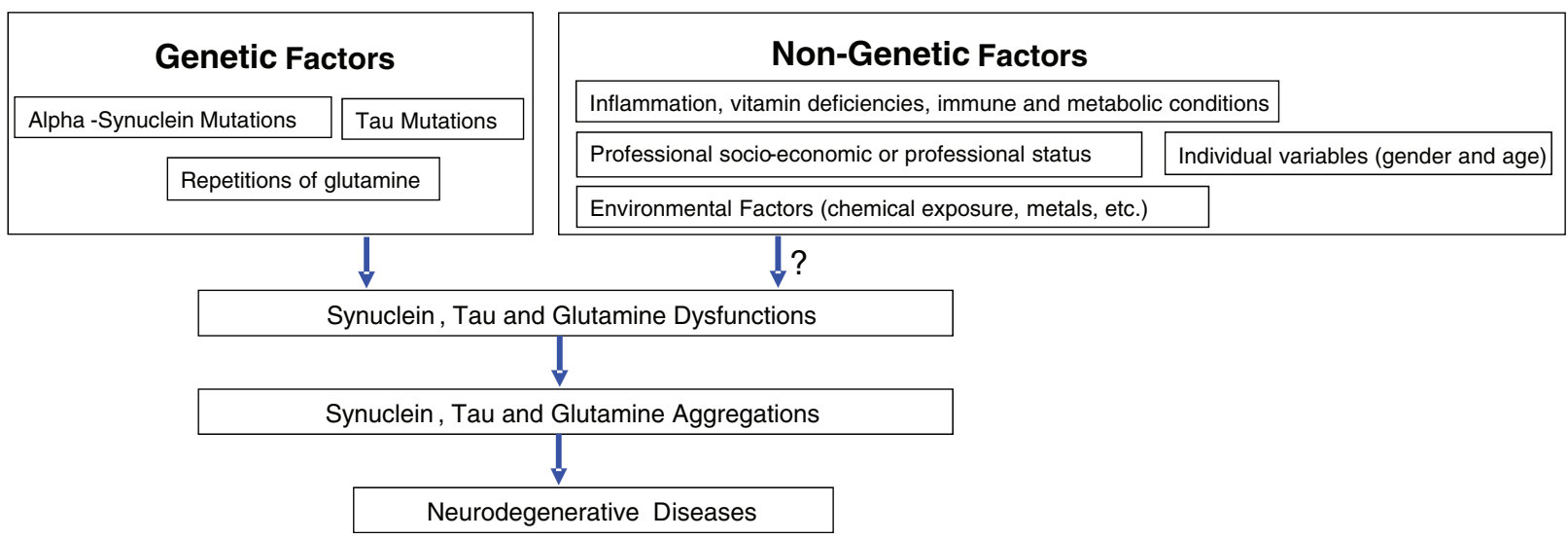

Fig. 3 - Spectrum of risk predisposing to common neurodegenerative diseases. This regrouping distinguishes genetic predisposition from non-genetic factors. 
$\mathrm{AD}$ is the most common neurodegenerative disease, affecting 20 to 25 million people worldwide, and is the fourth leading cause of death in industrialized countries. Its prevalence rate is around $7 \%$ in those aged $\geq 65$, with the doubling of risk for every 5 years after age 65 . Some studies of AD suggest that the gene encoding spondin 1 (SPON1) may be associated with the differential rate of cognitive decline in $A D$ patients $[14,15]$.

$\mathrm{PD}$ is the second most common neurodegenerative disease associated with intraneuronal filamentous inclusions made up of aggregated alpha-synuclein in Lewy bodies and dystrophic neurites. It affects between six and seven million people around the world. Some rare familial forms of PD are caused by mutations of the gene for alpha-synuclein [8].

DLB is the second most common neurodegenerative dementia after $\mathrm{AD}$, and the second most common synucleinopathy after PD, FTD represents $5-10 \%$ of all dementias, including Pick's disease. These disorders are related to mutations of the tau gene in familial FTD and parkinsonism linked to chromosome 17 (FTDP-17). For most of these diseases, the definitive diagnosis can only be made by pathological examination of brain tissue.

The primary neurodegenerative diseases that lead to a dementia syndrome have a number of common characteristics and constitute a consistent set of features:

- the neurodegenerative process is slow and can take 20 years before the onset of its first clinical manifestations, making it difficult to precisely date the beginning of the disease or even the start of the first clinical signs;

- the brain damage is irreversible, so the clinical signs persist over time;

- the symptomatology and all clinical signs of the dementia are important;

- the course of the illness trends towards a loss of independence.

\section{Conclusion}

There is accumulating evidence that most neurodegenerative diseases are due to deposits of aberrantly misfolded and aggregated brain proteins. Pathologies with a primary degenerative process mainly include $A D, P D, D L B, A L S, H D, F T D$ and prion diseases. $\mathrm{AD}$ is characterized by degeneration of neuronal cells due to filamentous neuronal inclusions, comprising the microtubule-associated protein tau in a hyperphosphorylated state; tau is the key constituent of neurofibrillary tangles and has a synergistic relationship with APP dysfunction.

The pathological accumulation of alpha-synuclein and Lewy bodies in the brains of PD patients (as postulated by Braak et al.) [16] could lead to the development of a predictable six-stage sequence of lesions, based on a stepwise pathological progression starting from the medullary nuclei and olfactory cortex. Implementation of such a staging strategy to categorize the degree of pathology in PD might then influence the patient's final diagnosis [16,17]. However, it has been shown that, in certain rare cases, there is no relationship between the Braak stage and clinical severity of PD [18].
The clinical syndrome of DLB seems to be directly related to Lewy body pathology and is often inversely related to the pathological lesions associated with AD. Thus, DLB neuropathological criteria could be useful for making a precise diagnosis. In particular, a visual semi-quantitative analysis could be used to differentiate DLB from PD in the brain: lesions in the latter have a distribution predominantly in the brainstem whereas, in the former, the distribution and frequency of Lewy bodies are likely be diffused throughout the neocortex. Imaging of the dopaminergic system using ${ }^{123}$ I-fluoropropyl-2-beta-carbomethoxy-3-beta(4-iodophenyl)nortropane (FP-CIT) single-photon emission computed tomography (SPECT) may represent an accurate method for differentiating DLB from other dementia syndromes [19].

Filamentous inclusions of tau protein have been identified in the brains of patients with FTD, and especially Pick's disease. This has been revealed to be due to mutations in the tau gene in familial forms of FTD, thus providing a direct link between tau protein dysfunction and dementia severity [8].

Finally, in MSA, which is less common than the other neurodegenerative pathologies and often confused with PD at the clinical level, the accumulation of misfolded alphasynuclein has been described, as well as multiple forms of neural degeneration similar to those found in PD and DLB [20].

In future, the development of strategies to elaborate new potentially therapeutic approaches and of further research aimed at the discovery of novel drugs for neurodegenerative diseases will require greater knowledge of the innate and adaptive immunological aspects of neurodegenerative diseases (such as those found in PD, AD and ALS) [21,22].

\section{Ethical approval}

The authors agree with ethical approval and informed written consent.

\section{Disclosure of interest}

The authors declare that they have no competing interest.

\section{R E F E R E N C E S}

[1] Hung CW, Chen YC, Hsieh WL, Chiou SH, Kao CL. Ageing and neurodegenerative diseases. Ageing Res Rev 2010;9(Suppl. 1):S36-46.

[2] Checkoway H, Lundin JI, Kelada SN. Neurodegenerative diseases. IARC Sci Publ 2011;163:407-19.

[3] Nieoullon A. Neurodegenerative diseases and neuroprotection: current views and prospects. J Appl Biomed 2011;9:173-83.

[4] Bertram L, Tanzi RE. The genetic epidemiology of neurodegenerative disease. J Clin Invest 2005;115(6): 1449-57.

[5] Brettschneider J, Del Tredici K, Lee VM, Trojanowski JQ. Spreading of pathology in neurodegenerative diseases: a focus on human studies. Nat Rev Neurosci 2015;16(2): 109-20. 
[6] Bourdenx M, Koulakiotis NS, Sanoudou D, Bezard E, Dehay B, Tsarbopoulos A. Protein aggregation and neurodegeneration in prototypical neurodegenerative diseases: examples of amyloidopathies, tauopathies and synucleinopathies. Prog Neurobiol 2015 [pii:S03010082(15)00077-5]

[7] Delacourte A. Tauopathies: recent insights into old diseases. Folia Neuropathol 2005;43(4):244-57.

[8] Goedert M, Spillantini MG. Synucleinopathies and tauopathies. [Chapter 47]In: Basic Neurochemistry, . 8th ed., 2012;829-43.

[9] Adams HH, de Bruijn RF, Hofman A, Uitterlinden AG, van Duijn CM, Vernooij MW, et al. Genetic risk of neurodegenerative diseases is associated with mild cognitive impairment and conversion to dementia. Alzheimers Demen 2015;11(11):1277-85.

[10] Stoyanova II. Ghrelin: a link between ageing, metabolism and neurodegenerative disorders. Neurobiol Dis 2014;72(Pt A):72-83.

[11] Parrón T, Requena M, Hernández AF, Alarcón R. Association between environmental exposure to pesticides and neurodegenerative diseases. Toxicol Appl Pharmacol 2011;256(3):379-85.

[12] Babenko O, Kovalchuk I, Metz GA. Epigenetic programming of neurodegenerative diseases by an adverse environment. Brain Res 2012;1444:96-111.

[13] Doty KR, Guillot-Sestier MV, Town T. The role of the immune system in neurodegenerative disorders: adaptive or maladaptive? Brain Res 2015;1617:155-73.
[14] Sherva R, Tripodis Y, Bennett DA, Chibnik LB, Crane PK, de Jager PL, et al. Consortium. The Alzheimer's disease neuroimaging initiative, and the Alzheimer's Disease Genetics Consortium. Genome-wide association study of the rate of cognitive decline in Alzheimer's disease. Alzheimer Demen 2014;10:45-52.

[15] Dunne TE. Alzheimer's disease: an overview. In: Encyclopedia of Mental Health, . 2nd ed., 2016;58-63.

[16] Braak H, Del Tredici K, Rüb U, de Vos RA, Jansen Steur EN, Braak E. Staging of brain pathology related to sporadic Parkinson's disease. Neurobiol Aging 2003;24:197-211.

[17] Alafuzoff I, Parkkinen L. Staged pathology in Parkinson's disease. Parkinsonism Relat Disord 2014;20:S57-61.

[18] Burke RE, Dauer WT, Vonsattel JP. A critical evaluation of the Braak staging scheme for Parkinson's disease. Ann Neurol 2008;64(5):485-91.

[19] Brigo F, Turri G, Tinazzi M. ${ }^{123}$ I-FP-CIT SPECT in the differential diagnosis between dementia with Lewy bodies and other dementias. J Neurol Sci 2015;359(1-2):161-71.

[20] Bendor JT, Logan TP, Edwards RH. The function of $\alpha-$ synuclein. Neuron 2013;79(6):1044-66.

[21] Rodrigues MC, Sanberg PR, Cruz LE, Garbuzova-Davis S. The innate and adaptive immunological aspects in neurodegenerative diseases. J Neuroimmunol 2014; 269(1-2):1-8.

[22] Kamsu-Foguem B, Tiako PF, Mutafungwa E, Foguem C. Knowledge-based modelling applied to synucleinopathies. European Geriatric Medicine 2015;6:381-8. 